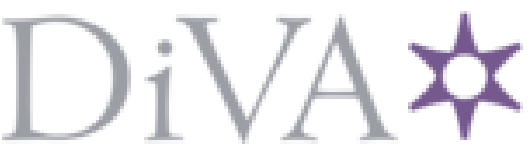

http://www.diva-portal.org

Preprint

This is the submitted version of a paper presented at 2018 IEEE OES Autonomous Underwater Vehicle Symposium.

Citation for the original published paper:

Rixon Fuchs, L., Gällström, A., Folkesson, J. (2018)

Object Recognition in Forward Looking Sonar Images using Transfer Learning In:

N.B. When citing this work, cite the original published paper.

Permanent link to this version:

http://urn.kb.se/resolve?urn=urn:nbn:se:kth:diva-250893 


\section{Object Recognition in Forward Looking Sonar Images using Transfer Learning}

\author{
Louise Rixon Fuchs \\ Robotics Perception and Learning, RPL \\ KTH - Royal Institute of Technology \\ Stockholm, Sweden \\ rixon@kth.se
}

\author{
Andreas Gällström \\ Sonar System Design \\ Saab Dynamics \\ Linköping, Sweden \\ andreas.gallstrom@saabgroup.com
}

\author{
John Folkesson \\ Robotics Perception and Learning, RPL \\ KTH - Royal Institute of Technology \\ Stockholm, Sweden \\ johnf@kth.se
}

\begin{abstract}
Forward Looking Sonars (FLS) are a typical choice of sonar for autonomous underwater vehicles. They are most often the main sensor for obstacle avoidance and can be used for monitoring, homing, following and docking as well. Those tasks require discrimination between noise and various classes of objects in the sonar images. Robust recognition of sonar data still remains a problem, but if solved it would enable more autonomy for underwater vehicles providing more reliable information about the surroundings to aid decision making. Recent advances in image recognition using Deep Learning methods have been rapid. While image recognition with Deep Learning is known to require large amounts of labeled data, there are data-efficient learning methods using generic features learned by a network pre-trained on data from a different domain. This enables us to work with much smaller domain-specific datasets, making the method interesting to explore for sonar object recognition with limited amounts of training data. We have developed a Convolutional Neural Network (CNN) based classifier for FLSimages and compared its performance to classification using classical methods and hand-crafted features.

Index Terms-AUV, CNN, Forward Looking Sonar, Object Recognition, Transfer Learning, Underwater, Data Efficient Learning
\end{abstract}

\section{INTRODUCTION}

Sonar is the preferred choice of sensor for imaging under water due to its long range and robustness to turbidity. Optical cameras are also popular sensors to use, but they are limited in range due to the attenuation of light in water. The different types of sonar have different strengths and weaknesses most importantly with regards to range, field of view and beam resolution.

Forward Looking Sonar (FLS) is, as the name suggests, a sonar mounted to look forward. There can be several variants of FLS. For the active case the principle of operation is that the sonar insonifies a scene with an acoustic wave [1]. The intensity of the acoustic return is then sampled as a function of range and bearing $(r, \theta)$. In the 2D case images built up from the acoustic returns are displayed in Cartesian coordinates. For

This work was supported by Stiftelsen for Strategisk Forskning (SSF) through the Swedish Maritime Robotics Center (SMaRC). an Autonomous Underwater Vehicle (AUV) the FLS is used to feed back information to control the vehicle.

An example of use for FLS on AUV is for monitoring a harbour. While operating underwater the AUV will have to respond to its environment. An example of information the AUV might need is: Are there any obstacles that the AUV needs to avoid? Or, is there a diver in this harbour area? For answering both these questions it is useful for the AUV to be able to recognize what it "sees" in its acoustic image from the FLS. Both resolution limitations and the noise characteristics of sonar make automatic classification of FLS images very challenging.

When we compare acoustic images produced by FLS to high resolution sonar system such as Synthetic Aperture Sonar (SAS) it is clearly more demanding to classify the lower resolution images. An example of what a SAS image looks like can be seen in Fig. 1 (left) where a tripod is depicted. An example of a FLS image of a boat hull is seen in Fig. 1 (right). The SAS image appears more photo-like than the FLS image.
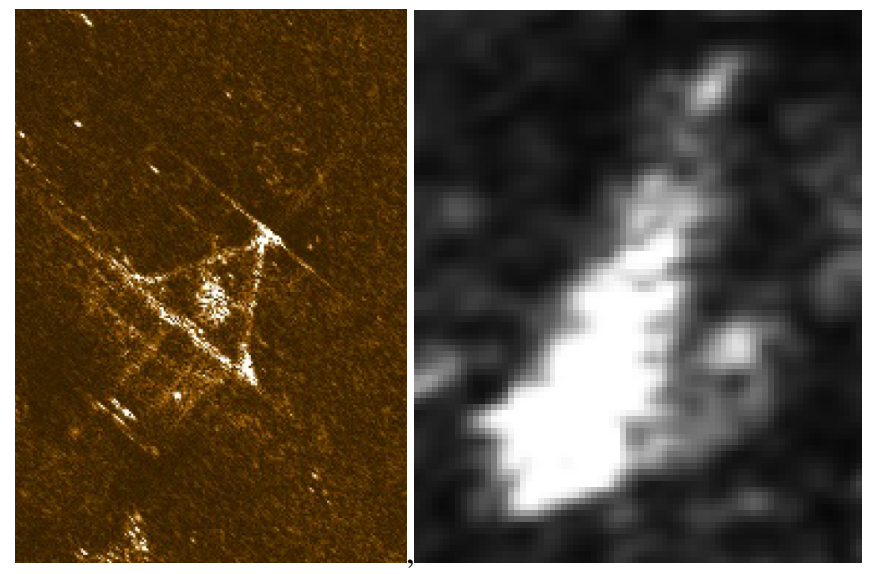

Fig. 1: Left: SAS image depicting a tripod, image from [2]. Right: FLS image of a boat hull from [3].

Being able to describe a scene in words as a "semantic 
map" has also proven useful to solve mobile robotics tasks for ground robots, see for example [4] and [5]. To create semantic information for our map, we need to detect and recognize our objects of interest. This work focuses on the object recognition task in FLS.

In this work, we explore the use of Deep Learning methods (DL) developed for computer vision to solve the acoustic image recognition problem in FLS images. Our target application is using FLS on an AUV to monitor an area such as a harbour. Collection and annotation of sonar data is difficult and often the amounts of training data is limited. This rules out the use of more data-hungry DL methods. One approach commonly used when limited amount of training data is available, is a method called transfer-learning. We will use a Convolutional Neural Network (CNN) trained on the ImageNet [6] database to extract generic features. Such features can be used to solve a variety of image recognition tasks as shown in for example [7].

Our goal here is to learn good features from limited amounts of data. This can avoid problems such as redundancy, bias or low discrimination power that can arise with hand-crafted features.

The contribution of this work is a new approach for object recognition in FLS images that makes use of features learned from a pretrained CNN. Our hypothesis is that CNNs designed for feature extraction for images can work well even for feature extraction for low resolution "blob-like" images.

We compare the new classifier with a classifier designed by using hand-crafted features [8]. We show that the DL approach of transfer learning can be a good alternative to using hand-crafted features. We also analyze how the size of the training set affects the test accuracy and find that it should be considered when designing a CNN-based classifier.

This paper is organized as follows. Section II provides a background on object recognition in sonar. Section III describes the method used and our system design with the new CNN-based classification. Section IV describes the experimental evaluation. Finally in section V we present our conclusions.

\section{BACKGROUND}

Object recognition in sonar images is a challenging problem. One of the challenges is that object recognition in sonar is not a view-invariant problem [9]. The returned backscatter of the sonar echo depends on several parameters for example, the frequency and source level of the sonar, the angle of incidence to the object of interest, etc. For more information see [10].

Another challenge is the lack of publicly available labelled training data. This has led to works aiming at creating synthetic sonar data, see for example [11] and [12].

Object recognition in sonar images is often performed using side-scan and synthetic aperture sonar systems, where one application is Computed Aided Detection and Computer Aided Classification systems [11], [13], [14], [15], [16].

The FLS is frequently used by AUVs as an object detection, and obstacle avoidance sensor [17], [18], [19], [20]. In [21],

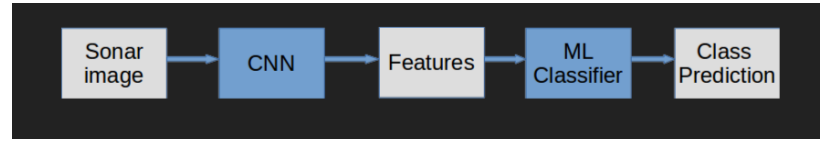

Fig. 2: Extraction of "bottleneck" features is shown. The term bottleneck refers to the fact that the network typically reduces the dimensionality from that of the image space to the feature space. In the context of transfer learning the hope is that similar features can be used for different learning tasks. This is tested by replacing the parts after the features are extracted with some other machine learning task. Transfer learning has the advantage of achieving high classification accuracy even in smaller datasets.

object detection in FLS images are analyzed using CNNarchitectures giving promising results. There has been some work in mosaicing FLS images to gain better recognition performance through obtaining higher image resolution [22]. A template based method for FLS has also been used for object recognition in [23].

Transfer learning in the context of image recognition is a method where a pretrained CNN is used as a "feature extractor" to extract useful features which are later used to train a Machine Learning (ML) classifier. In Fig. 2 the principle of extracting bottleneck features for classification is shown. An image of an object is fed through a pretrained CNN which extracts a vector of "bottleneck"-features. The bottleneckfeatures are then processed by a ML-classifier which outputs the class prediction of the object. In our case we are also investigating whether the features learned on one domain, photos, will transfer to a totally different domain and sensor, FLS.

Transfer learning can also employ a method of fine-tuning. The idea is to retrain or so called fine-tune some of the layers of the neural network and update its weight parameters. In this way, parts of the old architecture can be kept for feature extraction in a transfer learning way, while parts of the architecture are updated to fit the new data.

Work published on transfer learning in sonar images is limited. In forward looking sonar there is to our knowledge only one work done so far [24], using an acoustic camera with frequency of $3 \mathrm{MHz}$. Some work has been done on transfer learning in SAS-images see for example ( [25] and [26]). The previous works use sonar images of high resolution and uses larger datasets, this motivates us to explore if classification using transfer-learning could work even for lower-resolution "blob-like" sonar images in a smaller dataset.

\section{Method And System Design}

We propose a novel system design for object recognition in FLS using a CNN as part of the classifier. Our system is partly based on previous work from dos Santos et al.(2017) ( [8]), where an approach for object classification in four steps including image enhancement, segmentation, description, and classification is proposed. In our work, we use their approach 
Sonar image [3]

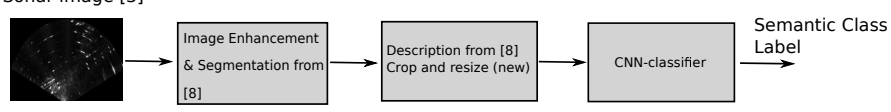

Fig. 3: Our new system with CNN-classifier.

up to the description step. In the description step we add a part where we crop out images from the FLS image, see Fig. 3 . Finally the cropped out images are processed by the CNN classifier.

The section below gives some explanation to the implementation using open source machine learning libraries.

\section{A. Implementation Machine Learning Library}

The CNN classifiers were implemented using Keras Sequential Model (Keras) [27] with Tensorflow [28] backend, and the scikit-learn library, a machine learning library for Python [29]. The Sequential Model in Keras is a linear stack of layers. To build a CNN that extracts bottleneck features, the first step is to create an empty sequential model. Next step is to import a $\mathrm{CNN}$-architecture from Keras application (in our case we choose ResNet50 [30] from which the last layer was removed).

Evaluation of the model's performance was done using standard functions from the scikit-learn library. For example the classification_report from sklearn.metrics to get the precision, recall and f1-scores for multiclass classification.

In the next section we give some information about the dataset and classes used for the object recognition task in this work.

\section{B. Dataset}

We use a ARACATI dataset from a marina environment [3]. Its acoustic images contains five labeled object classes, namely: boat-hulls, poles, fish, stones and swimmers. In total the dataset contains 531 labeled segments. The same dataset was also used by [8], to which we later compare our results in section $\mathrm{V}$.

The dataset was captured by a Blueview FLS with a frequency of $900 \mathrm{kHz}$. The range was configured to $30 \mathrm{~m}$.

An example of what the sonar images for the different classes looks like can be seen in Fig. 4. It is important to note that the images shown here have been cropped out from the FLS scan and re-sized to the same image size of $224 \times 224 \times 3$ pixels.

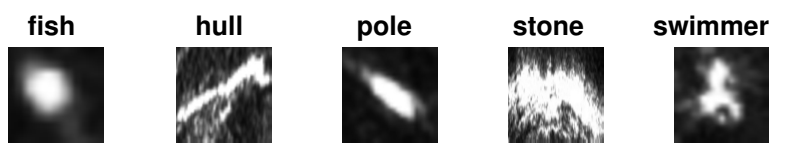

Fig. 4: Sonar images from the five classes.

\section{Creating images from segments}

The FLS images have been segmented, that means the objects of interest has been separated from the background. The segmented objects has then been described by Gaussians over their locations in the image. These steps were performed by the open source acoustic imaging tool [8]. A full FLS scan with the Gaussians marked in red is seen in Fig. 5.

In the original work, information about each segment is computed and then used as "hand-crafted" features. In total a vector of 10 features was computed. The features are: width, height, inertia ratio, std intensity, mean intensity, area, convex hull area, convexity, perimeter and pixel count. For details on how to compute the features we refer to their work.

Instead of computing information about the segments we choose to crop out images of them. The cropped out segments are then re-sized to $224 \times 224 \times 3$ pixels.

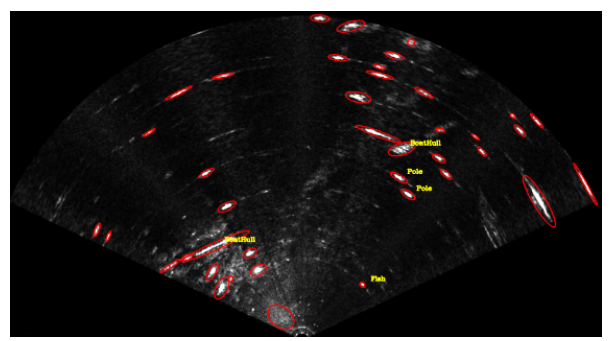

Fig. 5: FLS segmentation full image from [8]

\section{Transfer learning}

Transfer learning is often used when the new dataset is small and different from the original dataset and the classes to be learned are novel. There are two major transfer learning scenarios. One scenario using an existing network such as a $\mathrm{CNN}$ as a fixed feature extractor. Bottleneck features are extracted from the network by taking away its last layer or layers and treating the inputs to these layers as features.

The classification is then done in that feature space where classes hopefully are easily discriminated. Popular types of classifiers are the Softmax and Support Vector Machine (SVM) classifiers [31], which must learn using the limited domain training data.

The second scenario is called fine-tuning and involves retraining a CNN classifier to learn a new set of classes by only training a subset of the network layers with the new dataset [32]. This second method is best suited to learning new classes from a similar type of image data.

We will try to leverage the huge ImageNet dataset, but this data is not particularly similar to our low resolution sonar images. Therefore it would seem that the bottleneck feature approach is the better method for our problem. Furthermore we chose ResNet50 [30] as the fixed feature extractor because it can be considered a state-of-the-art architecture for feature extraction in transfer learning for image recognition. ResNet is a deep residual network architecture which gained attention in 2015 [30]. It is built up from stacking several subsections of residual blocks, which have skip connections. The skip connections prevents the gradient from vanishing and makes it possible to train deeper networks with more layers. We use ResNet50 which is a ResNet architecture with 50 residual 
blocks. The features extracted from ResNet50 are then used to train our classifiers.

\section{E. CNN-Classifier Design}

The CNN architecture used for both our classifiers is ResNet50 where the last layer has been removed. This architecture is used to extract bottleneck features for both CNNSoftmax and CNN-SVM. The sections that follows gives a short design description of each of the classifiers.

1) CNN-Softmax: For this version of the classifier the first layers of ResNet50 are "frozen", and a Softmax is added to the last layer, which is then trained on our new sonar data in form of bottleneck features extracted from the "frozen" layers. The Softmax layer is trained with the loss function categorical cross entropy.

2) $C N N-S V M$ : In the second classifier the bottleneck features are used to fit a SVM. The SVM is implemented with a one-vs-one scheme which is a combination of binary classifiers to build a multi-class classifier.

\section{EXPERIMENTAL EVALUATION}

Two types of experiments where made:

- Evaluation of the CNN-classifier models looking at the outputs from the two systems.

- Evaluation of how the size of the training set affects the overall classification accuracy for balanced datasets.

The evaluation metrics used where accuracy, precision, recall and f1-score.

To evaluate how well the $\mathrm{CNN}$-classifiers generalized to new data, K-Fold cross validation was used. It works in the way that each dataset is randomly partitioned into $\mathrm{k}$ smaller datasets of equal size. One of the subsamples is then used as the testing data, and the remaining k-1 subsamples are used for training data. The cross validation procedure is repeated $\mathrm{k}$ times. Each subsample is used exactly once as test data. In the end we take the average of the test accuracy from the $\mathrm{k}$ runs. For all our evaluation we use $\mathrm{k}=5$, which means we use $80 \%$ of the data for training and $20 \%$ for testing.

Equations for the evaluation metrics are given in (1)-(4), where $\mathrm{TP}=$ True Positive, $\mathrm{TN}=$ True Negative, $\mathrm{FN}=$ False negative, $\mathrm{FP}=$ False Positive.

$$
\begin{gathered}
\text { Accuracy }=\frac{\mathrm{TP}+\mathrm{TN}}{\mathrm{TP}+\mathrm{FP}+\mathrm{FN}+\mathrm{TN}} \\
\text { Precision }=\frac{\mathrm{TP}}{\mathrm{TP}+\mathrm{FP}} \\
\text { Recall }=\frac{\mathrm{TP}}{\mathrm{TP}+\mathrm{FN}} \\
\text { F1-score }=\frac{2 \times \text { Recall } \times \text { Precision }}{\text { Recall }+ \text { Precision }}
\end{gathered}
$$

\section{A. Object Recognition in unbalanced dataset}

For this evaluation an unbalanced dataset was used, see Table I. The dataset is unbalanced in the sense that it contains a different number of samples for the different classes. The size of the dataset is a little bit smaller but of comparable size to the unbalanced dataset in [8], which allows us to compare our results. The evaluation metrics used are precision, recall, and the F1-score (the harmonic average between precision and recall)

TABLE I: Smaller unbalanced dataset

\begin{tabular}{lllll}
\hline Class ID & Class Name & Total & Train & Val \\
\hline 0 & Fish & 80 & 60 & 20 \\
1 & Hull & 60 & 50 & 10 \\
2 & Pole & 240 & 190 & 50 \\
3 & Stone & 100 & 80 & 20 \\
4 & Swimmer & 30 & 20 & 10
\end{tabular}

The following section describes the evaluation of the CNNSoftmax classifier performance.

1) CNN-Softmax: The model is first cross-validated using stratified k-fold. Stratified k-fold means that the proportion of class labels area kept balanced between the different folds. It is trained with sgd (stochastic gradient descent) for 300 epochs. The training loss and the accuracy updated for each epoch is shown in Fig. 6, where we can see that the training loss converges. The result from the cross-validation evaluation gives an accuracy of $90 \%(+/-4 \%)$. The values within the parenthesis is the $95 \%$ confidence interval. The resulting precision, recall and F1-score per class label is presented in Table II.

\section{TABLE II: Result for CNN-Softmax}

$\begin{array}{lllllll} & \text { Total } & \text { Fish } & \text { Hull } & \text { Pole } & \text { Stone } & \text { Swimmer } \\ \text { Precision [\%] } & 98 & 100 & 83 & 98 & 100 & 100 \\ \text { Recall [\%] } & 97 & 90 & 100 & 100 & 100 & 90 \\ \text { F1 [\%] } & 97 & 95 & 91 & 99 & 100 & 95\end{array}$

The normalized confusion matrix for the experiment is shown in Fig. 7 (left). The diagonal of the matrix holds the results from recall or true positive rate, which is the proportion of actual positives that were correctly classified, the results from recall are also found in Table II. From the matrix we find that hulls, poles and stones have a perfect recall score of $100 \%$. The fish class gets confused for belonging to the hull or pole class, while the swimmer class get confused as hull.

2) $C N N-S V M$ : We first run a k-fold cross-validation. The result from this is an accuracy of $88 \%(+/-7.35 \%)$ the values in the parentheses indicates the $95 \%$ confidence interval.

Training with the smaller unbalanced dataset gives the results in Table III.

\section{TABLE III: Result for CNN-SVM}

$\begin{array}{lllllll} & \text { Total } & \text { Fish } & \text { Hull } & \text { Pole } & \text { Stone } & \text { Swimmer } \\ \text { Precision [\%] } & 89 & 94 & 83 & 84 & 91 & 100 \\ \text { Recall [\%] } & 88 & 85 & 50 & 98 & 100 & 60 \\ \text { F1 [\%] } & 87 & 89 & 62 & 91 & 95 & 75\end{array}$



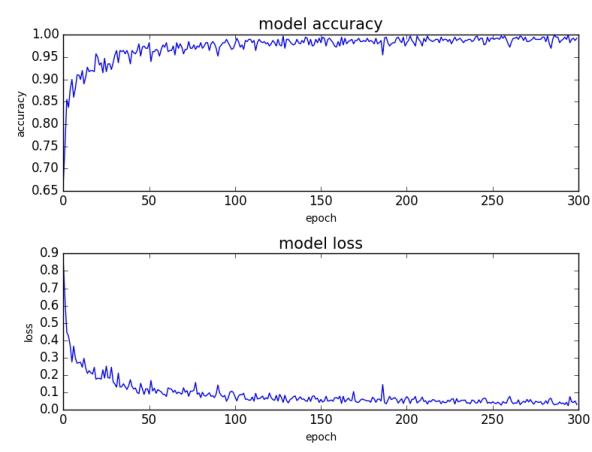

Fig. 6: Accuracy and Loss history curves for training CNNSoftmax

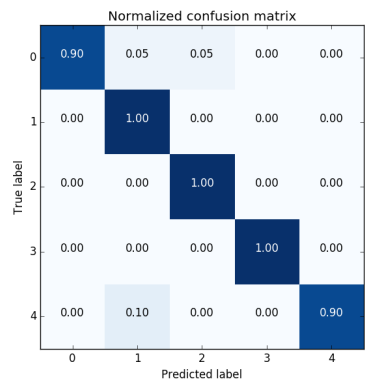

(a) CNN-Softmax

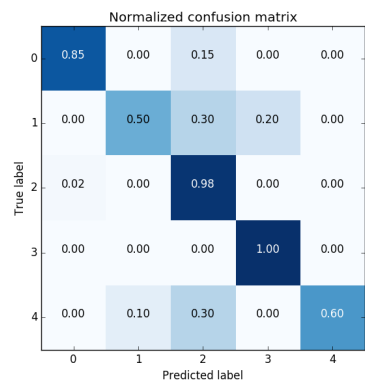

(b) CNN-SVM
Fig. 7: Normalized confusion matrices. The labels stands for the classes Fish=0, Hull=1, Pole=2, Stone=3, Swimmer=4.

The normalized confusion matrix for the experiment is shown in Fig. 7 (right). As before the diagonal of the matrix shows the recall of the classifier. This time only the stone class has a perfect recall score. The worst recall score is for the hull class, which is confused as pole and stone. Since boat-hulls are bigger than poles the classification algorithm would probably benefit from taking the size of the objects into account. One improvement to avoid such failures as classifying a hull as a pole could be to add a scale parameter to the classifier.

\section{B. Number of training samples and classification accuracy}

The training set sizes effect on the test accuracy was also explored. For this test three classes from the dataset were used: boat-hulls, poles and stones. For the training set we used a maximum of 100 samples per class. When not sufficient samples per class could be obtained from the original dataset the data was augmented, by adding replicated versions of images by vertically flipping the original images. Vertically flipping the sonar image ensures that the object's shadow (if present) does not appear to be in the front of the object. For evaluating this, k-fold crossvalidation was used for CNNSVM. For CNN-Softmax the evaluation was performed using crossvalidation with stratified k-fold. The CNN-Softmax was trained for 300 epochs. The results from this experiment is presented in Table IV. In the table the minimum, average, and maximum values for the classification accuracy are given.
TABLE IV: Classification accuracy vs number of training samples per class

\begin{tabular}{|c|ccc|ccc|}
\hline & \multicolumn{3}{|c|}{ CNN-Softmax } & \multicolumn{3}{c|}{ CNN-SVM } \\
\hline Samples no & Min & Avg & Max & Min & Avg & Max \\
\hline 5 & $67 \%$ & $80 \%$ & $100 \%$ & $33 \%$ & $73 \%$ & $100 \%$ \\
\hline 10 & $67 \%$ & $87 \%$ & $100 \%$ & $83 \%$ & $97 \%$ & $100 \%$ \\
\hline 20 & $92 \%$ & $93 \%$ & $100 \%$ & $92 \%$ & $98 \%$ & $100 \%$ \\
\hline 30 & $89 \%$ & $94 \%$ & $100 \%$ & $89 \%$ & $94 \%$ & $100 \%$ \\
\hline 40 & $83 \%$ & $93 \%$ & $100 \%$ & $88 \%$ & $94 \%$ & $100 \%$ \\
\hline 50 & $83 \%$ & $91 \%$ & $97 \%$ & $87 \%$ & $92 \%$ & $100 \%$ \\
\hline 60 & $89 \%$ & $94 \%$ & $100 \%$ & $83 \%$ & $91 \%$ & $97 \%$ \\
\hline 70 & $88 \%$ & $94 \%$ & $98 \%$ & $88 \%$ & $94 \%$ & $98 \%$ \\
\hline 80 & $90 \%$ & $95 \%$ & $100 \%$ & $92 \%$ & $93 \%$ & $96 \%$ \\
\hline 90 & $91 \%$ & $96 \%$ & $98 \%$ & $90 \%$ & $93 \%$ & $96 \%$ \\
\hline 100 & $87 \%$ & $94 \%$ & $98 \%$ & $93 \%$ & $94 \%$ & $95 \%$ \\
\hline
\end{tabular}

These results indicates that CNN-based classification for FLS-data can be a useful alternative to using hand-crafted features.

\section{CONClusion}

A CNN-based classification system for FLS images that uses transfer learning and pretrained CNNs to extract bottleneck features has been presented and evaluated. The results support that transfer learning can be an alternative to using hand-crafted features designing object recognition systems for FLS. This supports our hypothesis that feature extraction through pre-trained CNNs can work well even for low resolution FLS images.

The results from the evaluation of CNN-Softmax and CNNSVM run on the smaller unbalanced dataset seems to support that CNN-Softmax gives overall better performance with a total F1-score of $97 \%$ see Table II compared to a total F1score of $87 \%$ for CNN-SVM see Table III.

From looking at Table IV we draw the conclusion that the number of training samples per class does affect the resulting classification accuracy for our experiment. We see that the classification accuracy for both classifiers drop significantly when we have less than 20 samples. Above 20 training samples per class the result does not change noticeably, which suggest that we do not seem to benefit from adding more training samples after a certain threshold.

The images cropped-out and re-sized in our system are captured of the objects from different distances and view-angles, which shows that we can handle many different sonar-views and distances. It would be interesting to see if compensating for the distance to the object and the objects sizes would lead to better performance of the system.

The results shows that CNN based classification for FLS object recognition is a promising method. We note however that classification in FLS images is inherently difficult. It can not be expected that any method will yield the type of accuracy seen in camera image classification. There will always be ambiguity in these sonar images.

\section{A. Comparison to hand-crafted features}

Our method can be compared to the classification results of the original work [8]. The hand-crafted features are computed 
TABLE V: Unbalanced 10D Feature Results from [8].

$\begin{array}{lllllll} & \text { Total } & \text { Pole } & \text { Boat } & \text { Stone } & \text { Fish } & \text { Swimmer } \\ \text { Result Hit (\%) } & 84.40 & 81.63 & 84.61 & 100 & 88.8 & 50\end{array}$

from segments described by Gaussian probabilistic functions. In total they compute a 10D vector of 10 features. The features are: width, height, inertia ratio, std intensity, mean intensity, area, convex hull area, convexity, perimeter and pixel count. For a better explanation of these features we refer to their work.

Their best result from an unbalanced dataset which contains the same five classes and is slightly bigger than ours (531 images instead of 510) is a best result hit of $84.40 \%$ in total for a SVM classifier with a linear kernel, see Table V. It is interesting to note that the stone class gets a perfect score for both the hand-crafted features and for our $\mathrm{CNN}$-systems. The swimmer class that performs worst for the hand-crafted features also perform worst for the CNN-SVM classifier. This is of course a function of the classes chosen and a different set of classes would perhaps confound stones with some stonelike class.

Our best total result on our unbalanced dataset was with CNN-Softmax giving a total recall of $97 \%$. Looking at our results from k-fold crossvalidation where we have $88 \%(+/-$ $7.35 \%)$ accuracy for CNN-SVM and 90\% (+/- 4\%) accuracy for CNN-Softmax we find that our results are comparable to their best result. This indicates that CNN-based classification for FLS-data can be a useful alternative to using hand-crafted features for classification.

\section{ACKNOWLEDGEMENT}

This project is financed by the Swedish Maritime Robotic Centre, a national centre funded by the Swedish Foundation for Strategic Research (SSF).

\section{REFERENCES}

[1] Hurts Vilarnau, "Forward-looking sonar mosaicing for underwater environments," 2014.

[2] Ekblad, "Korrelering mellan optiskt och akustiskt avbildade objekt på havsbotten,” Linköpings universitet, Institutionen för systemteknik, Datorseende, 2013.

[3] "Acoustic Images of Dataset Aracati," https://goo.gl/mwd4gj, accessed: 2017-05-29.

[4] Kostavelis and Gasteratos, "Semantic mapping for mobile robotics tasks: A survey," Robotics and Autonomous Systems, vol. 66, no. C, pp. 86$103,2015$.

[5] Blodow et al., "Autonomous semantic mapping for robots performing everyday manipulation tasks in kitchen environments," in IEEE International Conference on Intelligent Robots and Systems, 2011, pp. 4263 4270.

[6] Deng et al., "ImageNet: A large-scale hierarchical image database," in 2009 IEEE Conference on Computer Vision and Pattern Recognition, June 2009, pp. 248-255.

[7] Razavian et al., "CNN Features off-the-shelf: an Astounding Baseline for Recognition," CoRR, vol. abs/1403.6382, 2014. [Online]. Available: http://arxiv.org/abs/1403.6382

[8] Dos Santos and Botelho, "Object Classification in Semi Structured Enviroment Using Forward-Looking Sonar." Sensors (Basel, Switzerland), 2017.

[9] $\mathrm{Cho}, \mathrm{Gu}$, and $\mathrm{Yu}$, "Robust sonar-based underwater object recognition against angle-of-view variation," Sensors Journal, IEEE, vol. 16, no. 4, pp. 1013-1025, 2016.
[10] Urick, Principles of underwater sound, 3rd ed. New York: McGrawHill, 1983.

[11] Pailhas, Petillot, and Capus, "High-resolution sonars: What resolution do we need for target recognition?" EURASIP Journal on Advances in Signal Processing, 2010. [Online]. Available: http://search.proquest.com/docview/856979529/

[12] Chen and Summers, "Deep neural networks for learning classification features and generative models from synthetic aperture sonar big data," Journal of the Acoustical Society of America, vol. 140, no. 4, pp. 3423 3423, 2016.

[13] Williams and Dugelay, "Multi-view sas image classification using deep learning." IEEE, 2016, pp. 1-9.

[14] Fei, Kraus, and Zoubir, "Contributions to automatic target recognition systems for underwater mine classification," vol. 53, 012015.

[15] Fandos and Zoubir, "Optimal feature set for automatic detection and classification of underwater objects in sas images," Selected Topics in Signal Processing, IEEE Journal of, vol. 5, no. 3, pp. 454-468, 2011.

[16] Myers and Fawcett, "A Template Matching Procedure for Automatic Target Recognition in Synthetic Aperture Sonar Imagery," Signal Processing Letters, IEEE, vol. 17, no. 7, pp. 683-686, 2010.

[17] Petillot, Tena Ruiz, and Lane, "Underwater vehicle obstacle avoidance and path planning using a multi-beam forward looking sonar," Oceanic Engineering, IEEE Journal of, vol. 26, no. 2, pp. 240-251, 2001.

[18] Karabchevsky, Braginsky, and Guterman, "Auv real-time acoustic vertical plane obstacle detection and avoidance," in 2012 IEEE/OES Autonomous Underwater Vehicles (AUV), Sept 2012, pp. 1-6.

[19] Kim and Yu, "Imaging sonar based real-time underwater object detection utilizing adaboost method," in 2017 IEEE Underwater Technology (UT), Feb 2017, pp. 1-5.

[20] Galceran et al., "A real-time underwater object detection algorithm for multi-beam forward looking sonar," IFAC Proceedings Volumes, vol. 45, no. 5, pp. 306-311, 2012.

[21] Livne A, Baruch A, "Thoughts on object detection using convolutional neural networks for forward-looking sonar." Int Rob Auto J. 2018;4(3).

[22] Ferreira et al., "Improving Automatic Target Recognition with Forward Looking Sonar Mosaics,' IFAC Proceedings Volumes, vol. 47, no. 3, pp. 3382-3387, 2014.

[23] Son-Cheol Yu et al., "Real-time 3d sonar image recognition for underwater vehicles." IEEE, 2007, pp. 142-146.

[24] Valdenegro-Toro, "Best Practices in Convolutional Networks for Forward-Looking Sonar Image Recognition," CoRR, vol. abs/1709.02601, 2017. [Online]. Available: http://arxiv.org/abs/1709.02601

[25] Warakagoda and Midtgaard, "Fine-tuning vs full training of deep neural networks for seafloor mine recognition in sonar images," 092017.

[26] McKay et al., "What's Mine is Yours: Pretrained CNNs for Limited Training Sonar ATR," CoRR, vol. abs/1706.09858, 2017. [Online]. Available: http://arxiv.org/abs/1706.09858

[27] Chollet et al., "Keras," https://github.com/fchollet/keras, 2015.

[28] Abadi et al., "TensorFlow: Large-scale machine learning on heterogeneous systems," 2015, software available from tensorflow.org. [Online]. Available: https://www.tensorflow.org/

[29] Pedregosa et al., "Scikit-learn: Machine learning in Python," Journal of Machine Learning Research, vol. 12, pp. 2825-2830, 2011.

[30] He et al., "Deep Residual Learning for Image Recognition," CoRR, vol. abs/1512.03385, 2015. [Online]. Available: http://arxiv.org/abs/1512.03385

[31] Tang, "Deep Learning using Support Vector Machines," CoRR, vol. abs/1306.0239, 2013. [Online]. Available: http://arxiv.org/abs/1306.0239

[32] Tajbakhsh et al., "Convolutional neural networks for medical image analysis: Full training or fine tuning?" Medical Imaging, IEEE Transactions on, vol. 35, no. 5, pp. 1299-1312, 2016. 